\title{
Diversifying clinical trials
}

\author{
Scientific common sense and social justice dictate that the safety and efficacy of new therapies must be tested \\ in the patient populations in need of treatment. Yet a recent study found that African Americans have been \\ dramatically underrepresented in US clinical trials for cancer drugs. Efforts to increase the participation of \\ minorities in clinical trials must become a priority for all drug developers.
}

A mong patient populations, the safety and efficacy of new treatments may vary with differences in sex, race, age or lifestyle, for example. Such variation in drug effects can only be detected by carrying out clinical trials on diverse patient populations, an aim that has a long history. In 1993, the US government passed a law that required the National Institutes of Health to ensure that federally funded clinical research prioritize the inclusion of women and minorities. More recently, in 2014, the US Food and Drug Administration (FDA) instituted an action plan that supports industry efforts at improving diversity in clinical trials. The following year, the FDA began publishing a 'Drug Trials Snapshot' that includes an analysis of the sex, race and age of clinical trial participants for every new drug approved.

Against this backdrop, the findings of a recent report (https://www.propublica. org/article/black-patients-miss-outon-promising-cancer-drugs) by the nonprofit news organization ProPublica, co-published with the news outlet STAT, are disheartening. This report analyzed Snapshot data from clinical trials for the 31 cancer drugs approved by the FDA since 2015 , comparing the demographics of these trials with the incidence of various cancers by race.

In trials for 24 of these 31 drugs, fewer than $5 \%$ of the patients were African American, despite the fact that African Americans make up $13.4 \%$ of the US population. For 18 of those drugs, the type of cancer targeted occurs in African Americans at least as frequently as in Americans of European descent. In trials for those types of cancer, on average only $4.1 \%$ of patients were African American. And in trials for four multiple myeloma drugs, only $5 \%$ of the participants, on average, were African American, whereas 14\% of people diagnosed with multiple myeloma are African Americans. A separate study by the FDA that analyzed clinical trials over a longer time frame similarly found that African-American patients make up only $4.5 \%$ of participants in multiple myeloma trials (Blood 130, 4352, 2017).

Many reasons have been put forward to explain the difficulties in enrolling African Americans in clinical trials. Chief among them is distrust of the medical establishment and fears of exploitation in medical research in this population (Am. J. Public Health 104, e16-e31, 2014). The infamous Tuskegee Study, in which the US Public Health Service deprived hundreds of African-American men of syphilis treatment so that researchers could study how the disease progressed, has cast a long shadow. In some communities, African Americans may also lack sufficient information regarding what clinical trials can offer and the safeguards in place to protect the privacy of their information. Beyond distrust and lack of information, substantial logistical hurdles can also impede fuller clinical trial participation. For example, some individuals may have limited access to the cancer centers that are the hubs of clinical studies, or they may lack the ability to take time off work to take part in a trial.

Recent findings may contribute to the wariness with which African Americans view medical research (Health Aff. (Millwood) 37, 1605-1614, 2018; https:// www.statnews.com/2018/10/01/africanamericans-clinical-trials). This report highlighted the high percentage (29\%), relative to their proportion of the United States population as a whole, of African Americans included over the last two decades in clinical trials in which patients were not required to give consent. These trials involved, for example, testing emergency medical procedures under conditions where the patient is physically incapable of giving consent. Such studies are often conducted in large medical centers in areas where African Americans may suffer disproportionately from the particular conditions being studied. Nevertheless, for a community in which the issue of consent resonates deeply, the high inclusion rate of African Americans in trials where patient consent was not obtained may undermine attempts to increase their participation in clinical trials.

These efforts must involve ongoing investment by those conducting clinical trials in engaging with and building trust in African-American communities. From a logistical perspective, placing study sites in areas of community outreach may ease the burdens that members of those communities face in order to participate in clinical trials.

New technologies that foster the decentralization of clinical trials may also offer opportunities for increased access for African Americans as well as other minorities not currently well represented in clinical trials. Several recently developed websites offer matchmaking between patients and clinical trials, enabling patients to find appropriate clinical trials no matter where the patients are physically located.

Recent efforts to generate comprehensive health, genomic and lifestyle profiles of the population, such as the All of Us initiative, have heightened awareness of issues of population diversity. Although race is a social construct that does not have a strict relationship to genetics, ensuring a representative sampling of the population across racial and ethnic boundaries is clearly a priority of such studies that requires high levels of engagement with historically neglected communities. With increasing awareness of the roles that genetics and lifestyle play in determining health and drug response, the time is now ripe to prioritize diversity in clinical trials.

Published online: 6 December 2018 https://doi.org/10.1038/s41591-018-0303-4 